Pixelized M-T-n CdTe detector coupled to Medipix2 readout chip

This article has been downloaded from IOPscience. Please scroll down to see the full text article.

2011 JINST 6 C01100

(http://iopscience.iop.org/1748-0221/6/01/C01100)

View the table of contents for this issue, or go to the journal homepage for more

Download details:

IP Address: 137.138.124.233

The article was downloaded on 27/07/2012 at 08:27

Please note that terms and conditions apply. 
$12^{\text {th }}$ INTERNATIONAL WORKSHOP ON RADIATION IMAGING DETECTORS,

JULY $11^{\text {th }}-15^{\text {th }} 2010$,

Robinson College, CAMBRIDGE U.K.

\title{
Pixelized M- $\pi$-n CdTe detector coupled to Medipix2 readout chip
}

\author{
J. Kalliopuska, ${ }^{a, 1}$ S. Nenonen, ${ }^{b}$ R. Penttilä, ${ }^{c}$ H. Pohjonen, ${ }^{a}$ L. Tlustos, ${ }^{d}$ A. Gädda, ${ }^{a}$ \\ J. Likonen, ${ }^{e}$ H. Andersson, ${ }^{b}$ P. Laakso ${ }^{c}$ and I. Vanttaja ${ }^{c}$ \\ ${ }^{a}$ VTT, Microsystems and Nanoelectronics, \\ Tietotie 3, Espoo, P.O. Box 1000, FI-02044 VTT, Finland \\ ${ }^{b}$ Oxford Instruments Analytical, \\ Nihtisillankuja 5, Espoo, PO. Box. 85, FI-02631, Finland \\ ${ }^{c}$ VTT, Laser Processing Applications, \\ Tuotantokatu 2, Lappeenranta, P.O. Box 17021, FI-53851, Finland \\ ${ }^{d}$ CERN, PH-ESE, \\ CH-1211, Genéva, Switzerland \\ ${ }^{e}$ VTT, Fusion and Plasma Technology, \\ Otakaari 3 K, Espoo, P.O. Box 1000, FI-02044 VTT, Finland
}

E-mail: Juha.Kalliopuska@vtt.fi

ABSTRACT: We have realized a simple method for patterning an M- $\pi-n$ CdTe diode with a deeply diffused pn-junction, such as indium anode on CdTe. The method relies on removing the semiconductor material on the anode-side of the diode until the physical junction has been reached. The pixelization of the p-type CdTe diode with an indium anode has been demonstrated by patterning perpendicular trenches with a high precision diamond blade and pulsed laser. Pixelization or microstrip pattering can be done on both sides of the diode, also on the cathode-side to realize double sided detector configuration.

The article compares the patterning quality of the diamond blade process, pulsed pico-second and femto-second lasers processes. Leakage currents and inter-strip resistance have been measured and are used as the basis of the comparison. Secondary ion mass spectrometry (SIMS) characterization has been done for a diode to define the pn-junction depth and to see the effect of the thermal loads of the flip-chip bonding process. The anode and cathode-sides of a $6 \times 6 \mathrm{~mm}^{2}$ diodes were patterned with a diamond blade and flip-chip bonded to the Medipix 2 readout chips. First imaging results with an X-ray source show reduced polarization effect and edgeless detector behavior for the anode-side patterned detector.

KEYWORDS: Solid state detectors; X-ray detectors; Pixelated detectors and associated VLSI electronics; Hybrid detectors

\footnotetext{
${ }^{1}$ Corresponding author.
} 


\section{Contents}

1 Introduction 1

2 Patterning processes and M- $\pi$-n CdTe diode structure 2

3 Electrical measurements $\quad 3$

4 Pixelization process $\quad 5$

5 Characterization with a radiation source 5

$\begin{array}{lll}6 & \text { Conclusions } & 7\end{array}$

\section{Introduction}

Patterning or pixelization of anode-side of an M- $\pi$-n CdTe detector, where the pn-junction is diffused into the detector bulk, is highly motivated for several reasons:

1. to obtain rectifying contact and lower leakage current levels than with the Schottky contacts,

2. to improve the spatial resolution by reading out the electrons instead of holes,

3. to improve the energy resolution by using the cathode-side as an entrance side for the radiation and read out of electrons,

4. to reduce the effects of polarization that is common in the M- $\pi$-n CdTe detector,

5. to be able to fabricate double sided M- $\pi$-n CdTe detector and

6. to obtain edgeless detector behavior in the CdTe detectors for realization of four-side buttable detector modules [1].

Applications for the M- $\pi$-n CdTe detectors with the above mentioned benefits are in the fields of security, electron microscopy, high energy physics in space and medical imaging. In the field of medical imaging, there is a high demand for a good quality polarization free direct conversion CdTe detectors for computed tomography and positron emission tomography.

It has been shown in [2], that the double-sided microstrip configuration for imaging detector can provide a fine spatial resolution and large detection areas with a suitable small amount of readout channels. The authors used Schottky-type CdTe detector due to problematic nature to segmenting the indium anode of an M- $\pi$-n CdTe detector. We have successfully reported in [3], a simple method to pattern the anode-side of the M- $\pi$-n CdTe detector. 


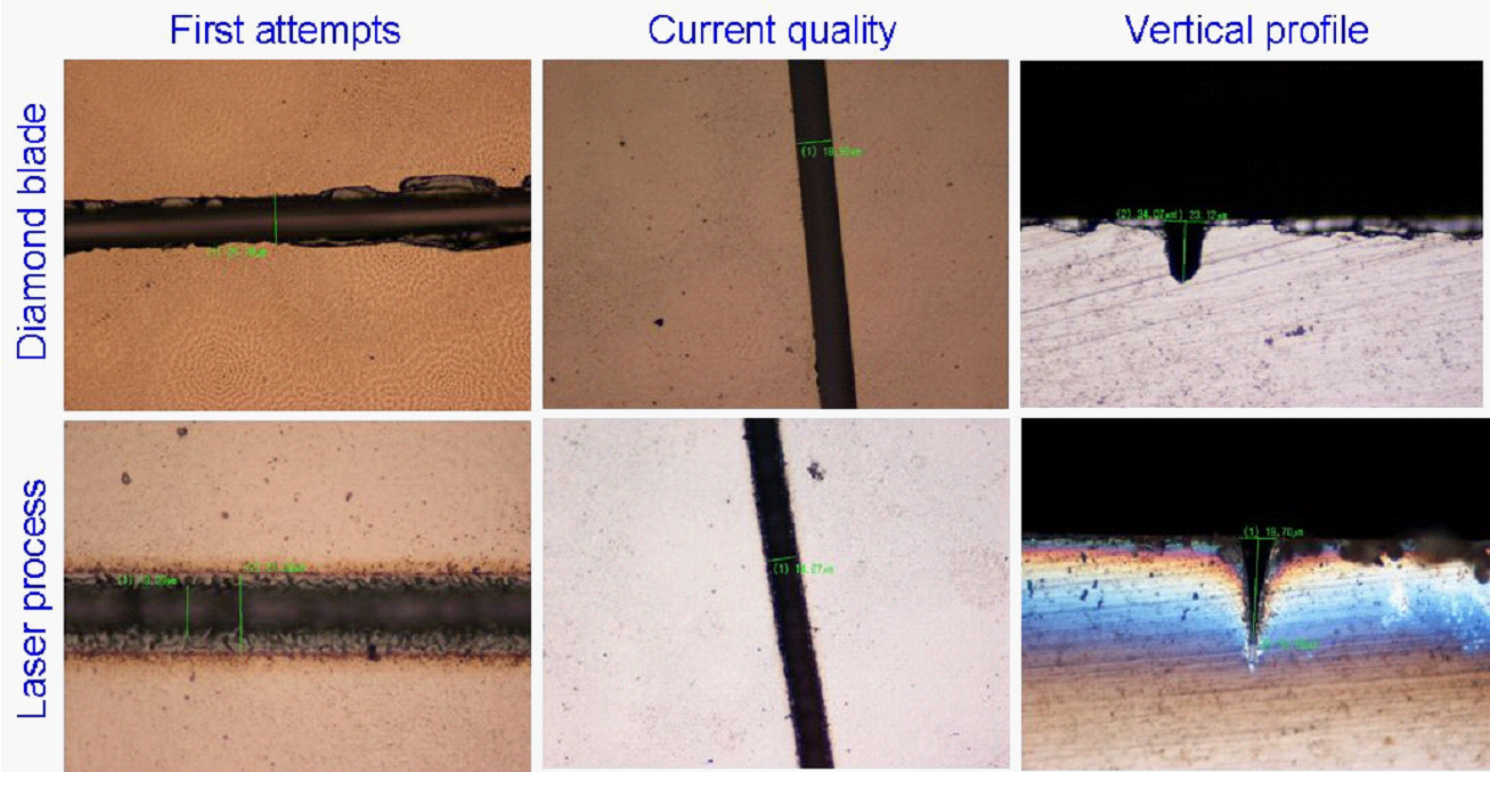

Figure 1. Microscope images of patterned CdTe diodes with the diamond blade and PS-laser.

Our method relies on removing the semiconductor material from the preferred locations until the pn-junction is reached. As the junction is penetrated, the separated segments become electrically isolated. The removal of the semiconductor material can be done by a pulsed laser, etching, diamond blade sawing, or by combining these methods. This article compares the patterning quality of the diamond blade saw and pulsed picosecond (PS) and femtosecond (FS) lasers.

\section{Patterning processes and M- $\pi$-n CdTe diode structure}

This section describes the results of visual inspection and Secondary Ion Mass Spectrometry (SIMS) characterization of patterned M- $\pi-n$ CdTe diodes. The patterning has been done on the anode-side of the diode with a diamond blade, FS- and PS-lasers. The pitch of the patterned trenches was 300 $\mu \mathrm{m}$ and the depths of the trenches were from $10 \mu \mathrm{m}$ to $40 \mu \mathrm{m}$ in $10 \mu \mathrm{m}$ steps. Five strips were patterned to each depth.

Figure 1 shows microscope images of top and side profiles of the diamond blade and laser patterned diodes. The diamond blade process results in U-shaped profile and the pulsed laser process leads in V-shaped profile. First attempts to pattern the CdTe diode with the diamond blade caused cracking of the crystal along the trenches. Currently this cracking has been removed as figure 1 illustrates. The first attempts of the pulsed laser processes (FS and PS) caused burning of the surface, but the current process yields no burning.

The $6 \times 6 \mathrm{~mm}^{2} \mathrm{M}-\pi-\mathrm{n}$ CdTe diodes were purchased from Acrorad Ltd. To determine the internal structure of the sample diodes, the SIMS characterization was performed. With SIMS alone, one is not able to determine the pn-junction depth directly, but it can be used to give more knowledge about the structure. For comparison we made the SIMS characterization for a diode used in [3]. Figures 2(a) and 2(b) show the ion isotope count rates with highlighted signals of Cd (114), Te (130) and In (115) of the new and old CdTe samples from [3], respectively. In the 


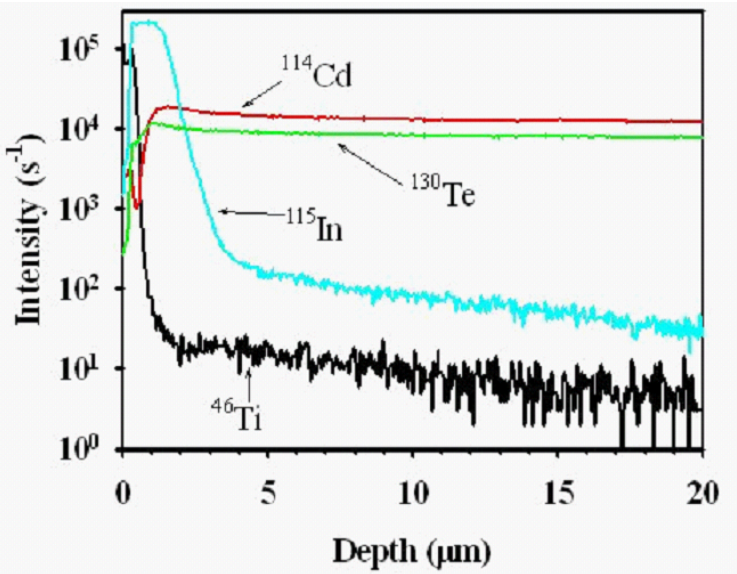

(a)

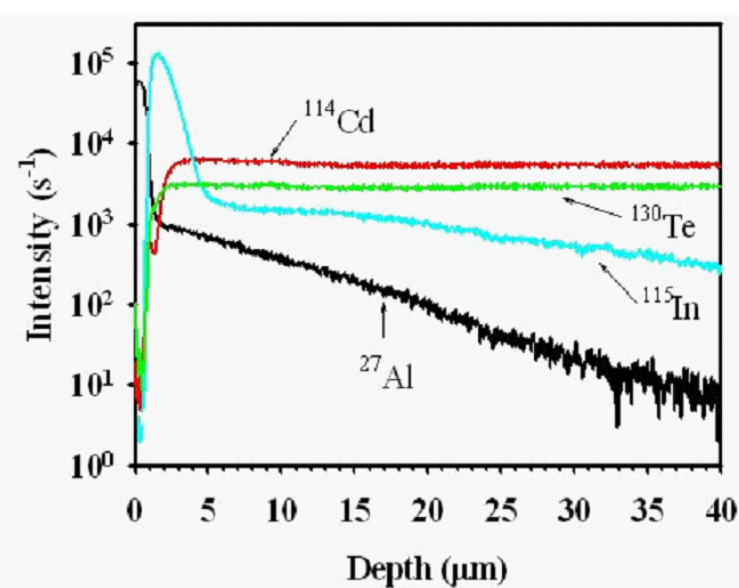

(b)

Figure 2. SIMS characterization results of (a) new and (b) old M- $\pi$-n CdTe diode samples.

old sample, the Indium count rate maintains almost constant for several micrometers before the junction is reached and decreases after that point. In the new sample, the thermal processes during the fabrication are well managed and the diffusion is in control resulting in the pn-junction depth below $5 \mu \mathrm{m}$.

\section{Electrical measurements}

The different patterning processes were compared electrically by measures of leakage current and inter-strip resistance. The measurements were done similarly as explained in [3].

Figure 3 shows the leakage current measurements of an un-patterned diode and the patterned diodes with different trench depths on the anode-side. The un-patterned diode has a leakage current of $2 \mathrm{nA} / \mathrm{cm}^{2}$ at $100 \mathrm{~V}$ reverse bias, where as the old sample diode, measured in [3], had $12 \mathrm{nA} / \mathrm{cm}^{2}$. The leakage current curves of $10 \mu \mathrm{m}$ deep trenches are peculiar due to bad inter-strip isolation, as later shown in figure 4. The leakage current levels are smallest for PS-laser process and are 2-5 $\mathrm{nA} / \mathrm{cm}^{2}$ at $100 \mathrm{~V}$ reverse bias. The FS-laser and diamond blade processes resulted in similar current levels of $10-35 \mathrm{nA} / \mathrm{cm}^{2}$ at $100 \mathrm{~V}$ reverse bias. The results indicate that the leakage current increases as a function of the depth of the trench. The leakage current measurements presented in [3] yielded leakage currents of $12-17 \mathrm{nA} / \mathrm{cm}^{2}$ at $100 \mathrm{~V}$ reverse bias for the diamond blade process.

Figure 4 shows the measured inter-strip resistances of the patterned CdTe diodes. The interstrip current was measured, as described in [3], and its inverse derivative is plotted. A reverse bias of $50 \mathrm{~V}$ was applied over the diode during the measurement to deplete the pn-junction side of the detector. After the strips are electrically isolated by the depletion region, the inter-strip resistance does not change significantly with the bias voltage.

Figure 4 shows that the inter-strip resistances of $10 \mu \mathrm{m}$ deep trenches are fairly poor having values of $0.1-10 \mathrm{M} \Omega$. This causes a poor electrical isolation and peculiar leakage currents as was discussed above. Clear differences were observed between the patterning methods with respect to the inter-strip isolation. The diamond blade process yielded very good isolation of 100-1000 


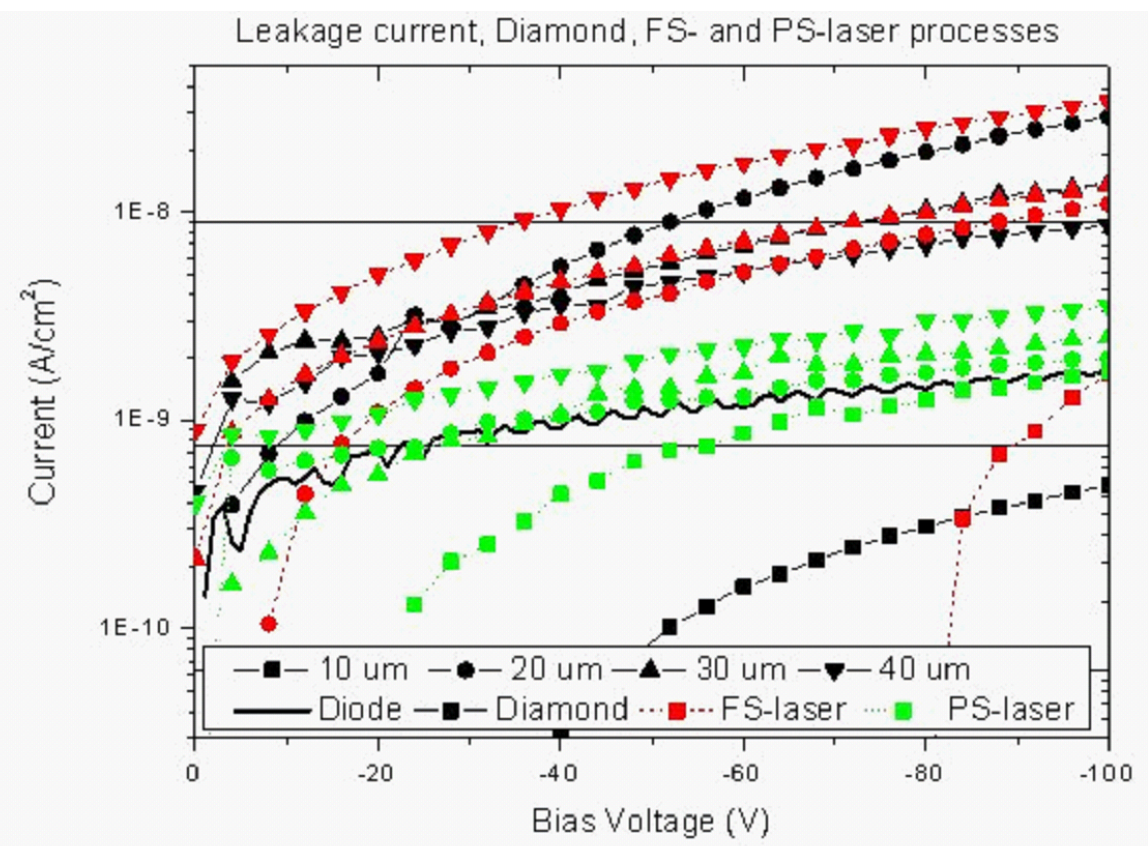

Figure 3. Measured leakage currents of the patterned CdTe diodes with different trench depths.

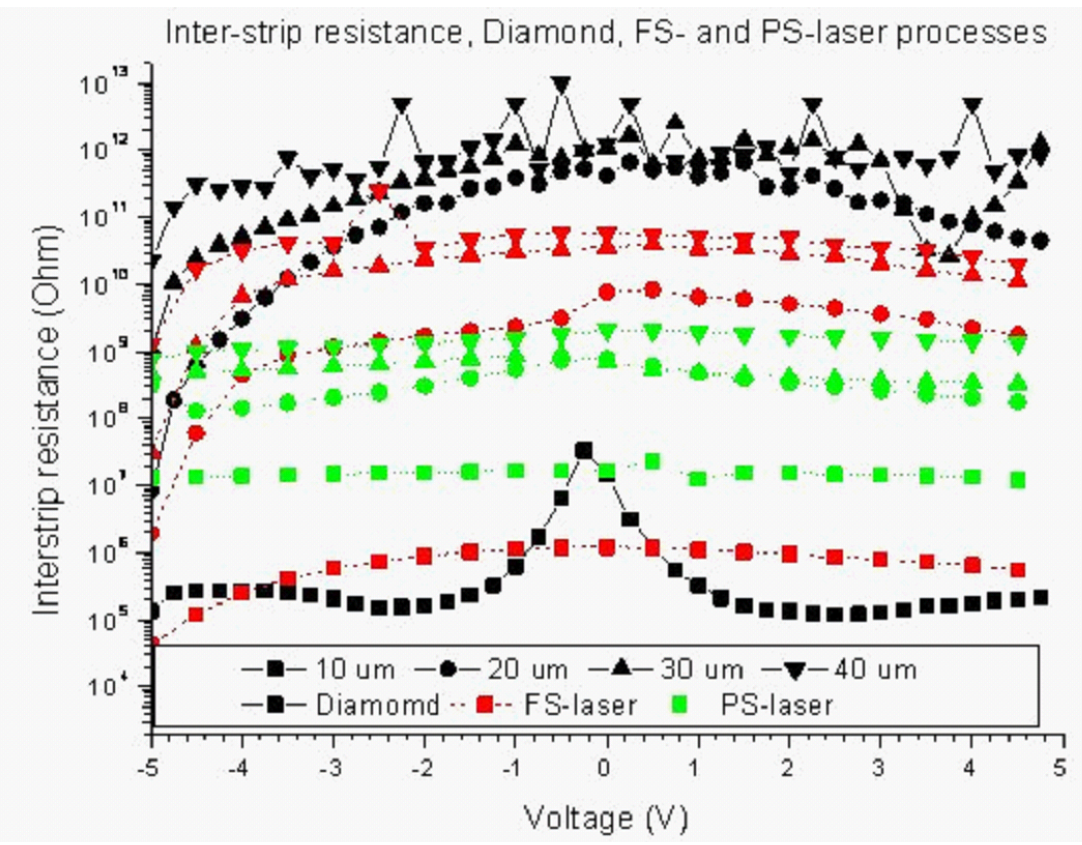

Figure 4. Measured inter-strip resistances of the patterned CdTe diodes.

$\mathrm{G} \Omega$. Values of $200 \mathrm{G} \Omega$ were obtained at best in [3]. A good isolation was obtained with the FSlaser process having values of 1-50 G $\Omega$. Even though the lowest leakage current performance was obtained with the PS-laser process, it still results in a fair electrical isolation of $0.3-1 \mathrm{G} \Omega$. 


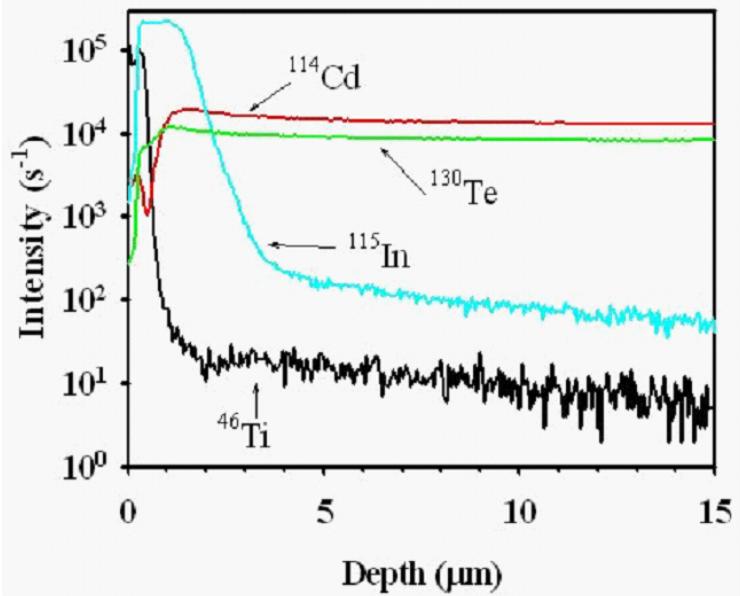

(a)

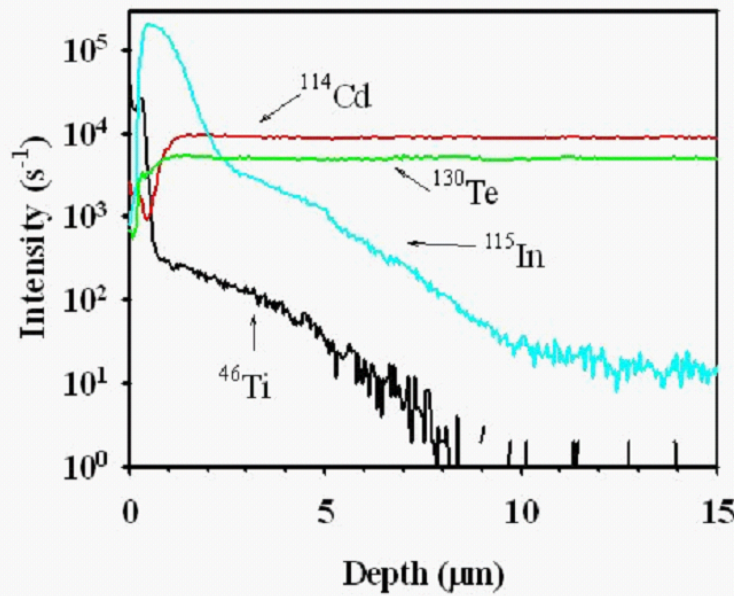

(b)

Figure 5. SIMS characterization of the M- $\pi$-n CdTe detector (a) before and (b) after the flip-chip bonding process.

\section{Pixelization process}

Two $6 \times 6 \mathrm{~mm}^{2} \mathrm{M}-\pi$-n CdTe diodes were pixelated, one from the anode and the other from cathodeside, by the diamond blade process. Perpendicular trenches of $10 \mu \mathrm{m}$ deep were patterned with a grid spacing of $55 \mu \mathrm{m} \times 55 \mu \mathrm{m}$. These detectors were flip-chip bonded to individual Medipix2 readout chips (ROCs) [4] with $\mathrm{Sn}-\mathrm{Pb}$ solder bumps. The conventional flip-chip process with the $\mathrm{Sn}-\mathrm{Pb}$ solders requires a high temperature cycle up to $230{ }^{\circ} \mathrm{C}$.

The effect of the thermal load of the flip-chip process to the depth of the pn-junction was studied with the SIMS characterization. The thermal cycle of the bonding process, which consists of $30 \mathrm{~s}$ at $185^{\circ} \mathrm{C}$ and $90 \mathrm{~s}$ at $230{ }^{\circ} \mathrm{C}$, was simulated for a diode. Figures 5(a) and 5(b) show the results of the SIMS before and after the bonding process. It can be observed that the n-type Indium has diffused towards the bulk of the p-type diode and the pn-junction has drifted from around $3 \mu \mathrm{m}$ to $6 \mu \mathrm{m}$.

\section{Characterization with a radiation source}

Figure 6(a) shows the polarization effects of the anode-side patterned detector assembly at different reverse bias voltage conditions during the exposure to Am-241 radiation source. The polarization effect leads to a drop in a count rate due to cancelation of the internal electric field of the detector and thus to a charge lost via recombination and trapping processes. In figure 6(a), the count rate drops and then saturates at reverse bias voltages below $500 \mathrm{~V}$. At $500 \mathrm{~V}$, the count rate remains almost constant during 3.5 hours of acquisition. If the polarization effect occurs, it can be recovered by quickly switching the bias off and on again.

Figs 6(b) show the Am-241 flood field images of the anode-side patterned detector assembly during the high count rate acquisition period at $300 \mathrm{~V}$. Image shows a good quality of flip-chip bonding as there are only few dead pixels. The high temperature flip-chip bonding process is the 


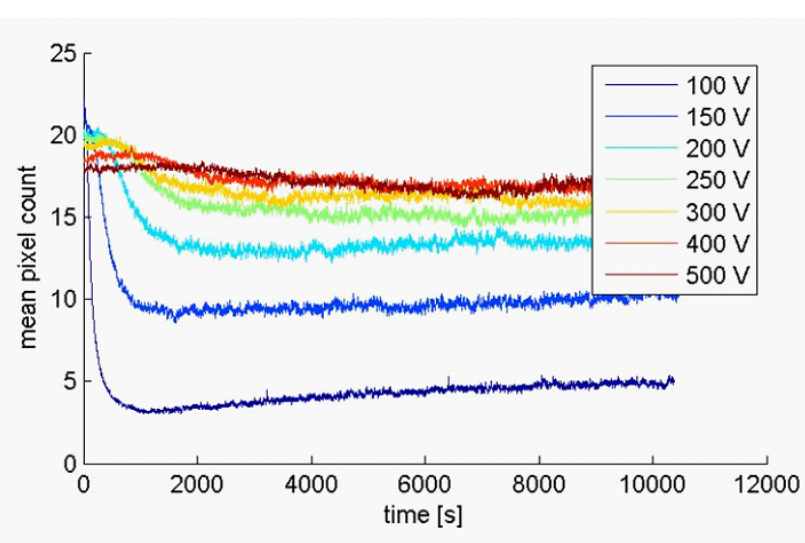

(a)

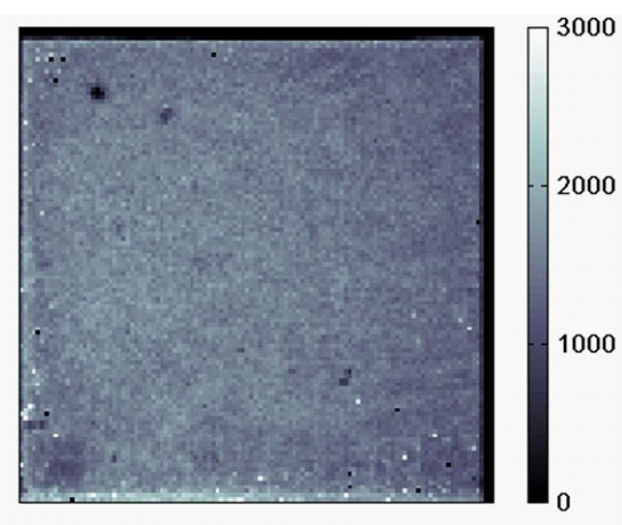

(b)

Figure 6. (a) Polarization of anode-side patterned pixel detector at different bias voltages. (b) Am-241 flood field images taken at $300 \mathrm{~V}$ reverse bias condition during the first $6 \mathrm{~min}$ of the measurement.

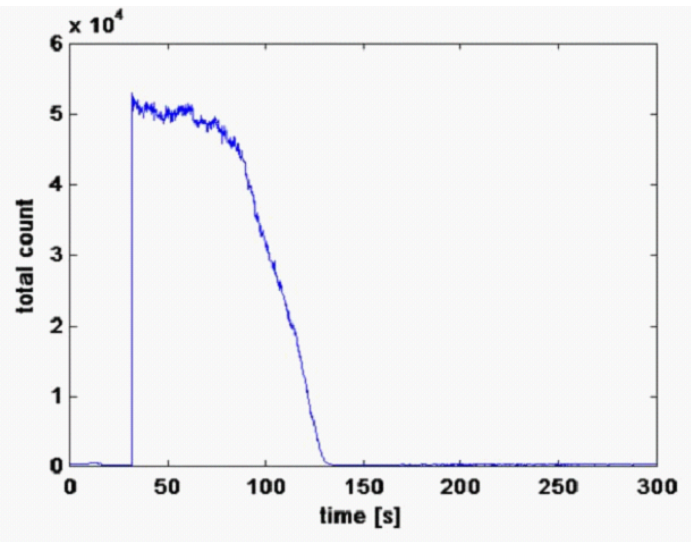

(a)

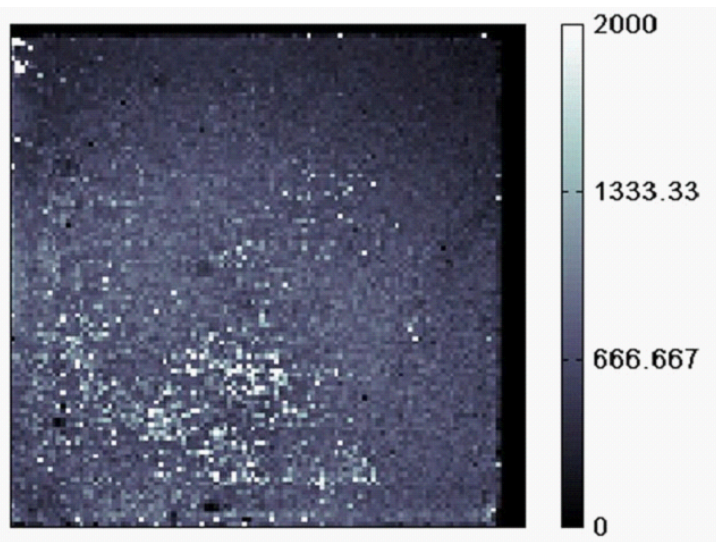

(b)

Figure 7. (a) Polarization of cathode-side patterned pixel detector at $300 \mathrm{~V}$ bias. (b) Am-241 flood field images taken at $300 \mathrm{~V}$ reverse bias condition during the first 6 min of the measurement.

expected cause of observed defects in the image. To overcome this issue, a low temperature solder technology with In-Sn bumps has been developed recently at VTT, as presented in [5].

In addition, figure 6(b) shows higher count rates close to the edges than at the center of the detector matrix. This might be an indication of a distorted electric field at a perimeter of the edges and signify an inherent edgeless behavior of the $M-\pi$-n CdTe pixel detectors. The cathodeside patterned detector, in figure 7(b), did not show this kind of behavior, as is expected. For reference, see article [6], where the similar behavior has been demonstrated with edgeless silicon pixel detectors.

Figure 7(a) shows the polarization effects of the cathode-side patterned detector assembly at $300 \mathrm{~V}$ reverse bias. The count rate begins to drop after $1 \mathrm{~min}$ and only few counts are received after 2 minutes of acquisition. Figs 7(b) show the Am-241 flood field images of the cathode-side 
patterned detector assembly during the high count rate acquisition period at $300 \mathrm{~V}$. The reason for a poor contrast in the image is the fast onset of the polarization and thus low number of counts per pixel. Several noise pixels can be observed at the image. This is caused by the peeling of the metal away from the pixels during the diamond blade process.

\section{Conclusions}

The article studied and compared the diamond blade, femtosecond (FS) and picosecond (PS) pulsed lasers processes on the anode-side of the M- $\pi$-n CdTe diode.The patterning method for the M- $\pi$-n CdTe diodes was published in [3]. The comparison was done by measures of leakage current and inter-strip resistance and the following numbers are given at $100 \mathrm{~V}$ reverse bias for the $6 \mathrm{~mm}$ long strips. The lowest leakage current levels of $2-5 \mathrm{nA} / \mathrm{cm}^{2}$ were measured with the PS-laser process but with a poor inter-strip isolation of $0.3-1 \mathrm{G} \Omega$. The FS-laser and the diamond blade processes resulted in similar leakage currents of $10-35 \mathrm{nA} / \mathrm{cm}^{2}$ with the inter-strip isolation of 1-50 $\mathrm{G} \Omega$ and $100-1000 \mathrm{G} \Omega$, respectively.

Two $6 \times 6 \mathrm{~mm}^{2} \mathrm{M}-\pi$-n CdTe pixel detector prototypes were patterned from the anode-and cathode-sides by using the diamond blade process and coupled to the Medipix 2 readout chip (ROC) for characterization. It was demonstrated by using the SIMS characterization that the low temperature flip-chip bonding process is required to avoid drifting of the pn-junction deeper into the detector bulk. Improved tolerance against the polarization effect was measured with the anode-side

patterned M- $\pi-\mathrm{n}$ CdTe pixel detector. The first X-ray source images revealed indications of the inherent edgeless behavior of the anode-side patterned $M-\pi$-n CdTe detectors. Further study is needed to understand the polarization effect and the edgeless detector behavior.

\section{Acknowledgments}

The authors of the paper would like to thank European Space Agency for funding the project with in their Innovation Triangle Initiative funding program.

\section{References}

[1] S. Vähänen et al., 3D Integration Activities at CERN, presented at VIPS 2010 Workshop.

[2] K. Nakazawa et al., A high energy resolution $4 \mathrm{~cm}$-wide double-sided silicon strip detector, Nucl. Instrum. Meth. A 573 (2007) 44.

[3] J. Kalliopuska et al., Patterning thick diffused junctions on CdTe, Nucl. Instrum. Meth. A 607 (2009) 98.

[4] X. Llopart et al., Medipix2, a 64k pixel readout chip with $55 \mu \mathrm{m}$ square elements working in single photon counting mode, IEEE Trans. Nucl. Sci. 49 (2002) 2279.

[5] H.Heikkinen et al., Indium-Tin Bump Deposition for the Hybridization of CdTe Sensors and Readout Chips, to be published in proceedings of IEEE $16^{\text {th }}$ Room Temperature Semiconductor Detector Workshop, Knoxville, Tennessee, U.S.A. (2010).

[6] J. Kalliopuska et al., Characterization of edgeless pixel detectors coupled to Medipix2 Readout Chip, Nucl. Instrum. Meth. A in press. 